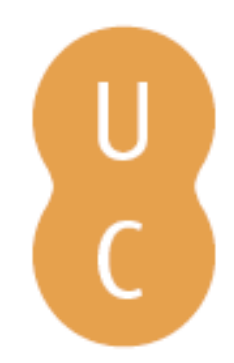

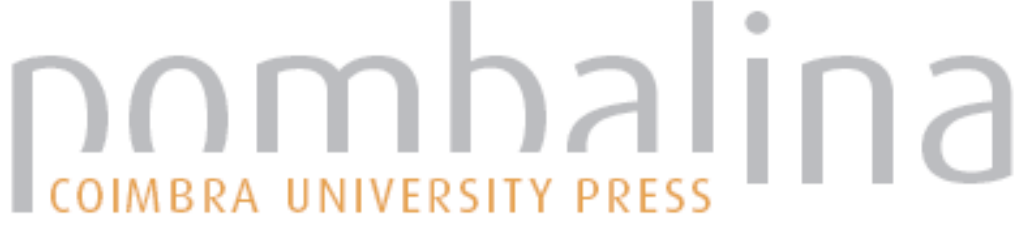

Nitratos nos solos e águas subterrâneas - perspetiva sobre a atualidade, tendências, implicações para a saúde ambiental e abordagens de redução de riscos
Autor(es):
Araújo, Rui; Castro, Ana Meira; Fiúza, António
Publicado por: $\quad \begin{aligned} & \text { Imprensa da Universidade de Coimbra; RISCOS - Associação } \\ & \text { Portuguesa de Riscos, Prevenção e Segurança }\end{aligned}$
URL persistente:
URI:http://hdl.handle.net/10316.2/34888
DOI:
DOI:http://dx.doi.org/10.14195/978-989-96253-3-4_87
Accessed : $\quad$ 26-Apr-2023 15:54:37

A navegação consulta e descarregamento dos títulos inseridos nas Bibliotecas Digitais UC Digitalis, UC Pombalina e UC Impactum, pressupõem a aceitação plena e sem reservas dos Termos e Condições de Uso destas Bibliotecas Digitais, disponíveis em https://digitalis.uc.pt/pt-pt/termos.

Conforme exposto nos referidos Termos e Condições de Uso, o descarregamento de títulos de acesso restrito requer uma licença válida de autorização devendo o utilizador aceder ao(s) documento(s) a partir de um endereço de IP da instituição detentora da supramencionada licença.

Ao utilizador é apenas permitido o descarregamento para uso pessoal, pelo que o emprego do(s) título(s) descarregado(s) para outro fim, designadamente comercial, carece de autorização do respetivo autor ou editor da obra.

Na medida em que todas as obras da UC Digitalis se encontram protegidas pelo Código do Direito de Autor e Direitos Conexos e demais legislação aplicável, toda a cópia, parcial ou total, deste documento, nos casos em que é legalmente admitida, deverá conter ou fazer-se acompanhar por este aviso.

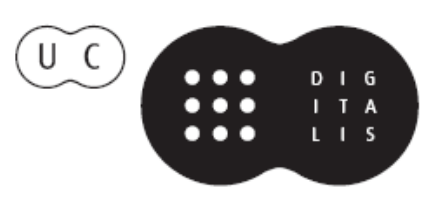



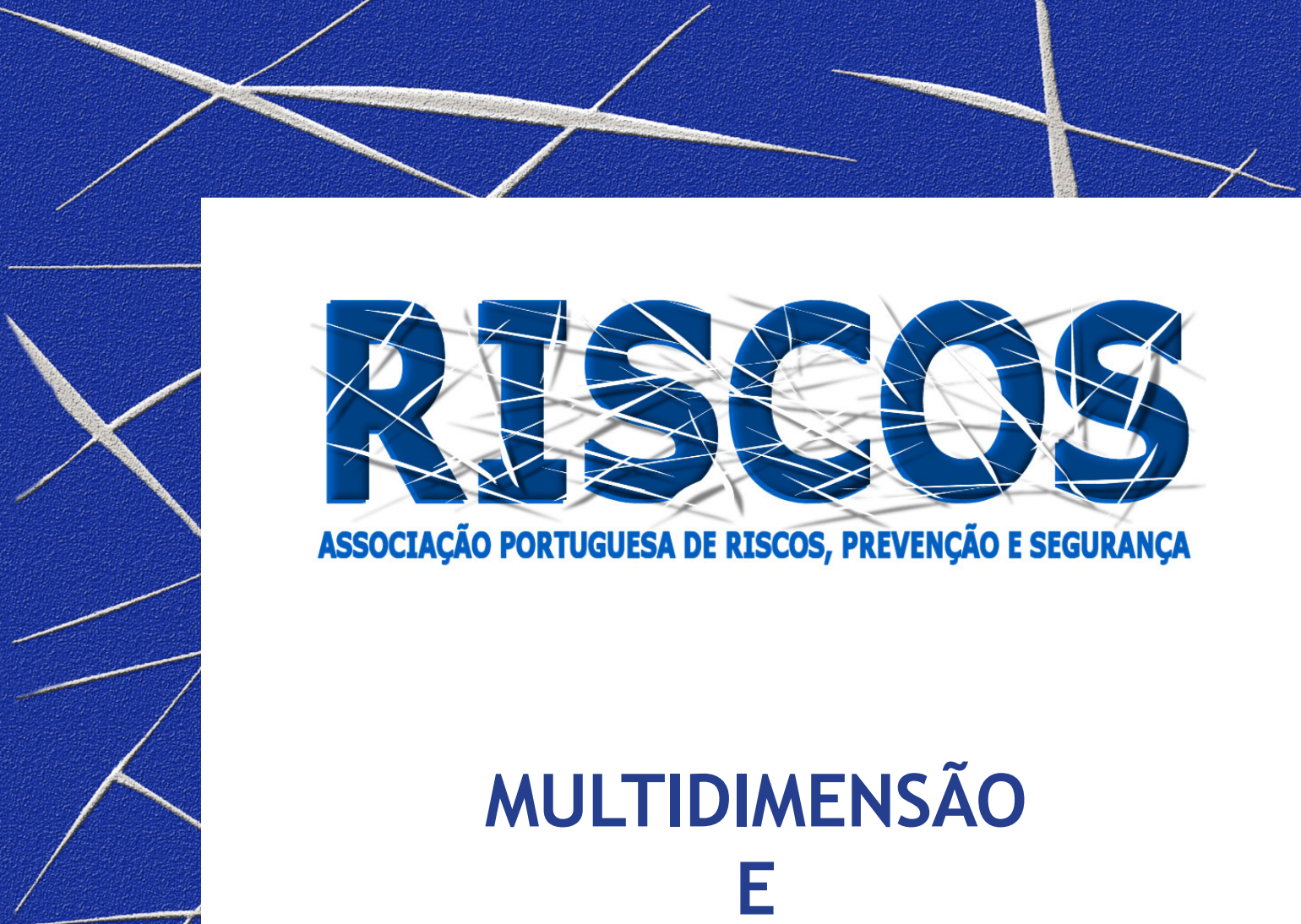

ASSOCIAÇÃO PORTUGUESA DE RISCOS, PREVENCCÃO E SEGURANÇA

MULTIDIMENSÃO

E
TERRITÓRIOS DE RISCO

III Congresso Internacional

I Simpósio Ibero-Americano

VIII Encontro Nacional de Riscos

Guimarães

2014 


\title{
NITRATOS NOS SOLOS E ÁGUAS SUBTERRÂNEAS - PERSPETIVA SOBRE A ATUALIDADE, TENDÊNCIAS, IMPLICAÇÕES PARA A SAÚDE AMBIENTAL E ABORDAGENS DE REDUÇÃO DE RISCOS
}

\author{
Rui Araújo \\ Faculdade de Engenharia, Universidade do Porto \\ demss09000758@fe.up.pt \\ Ana Meira Castro \\ Departamento de Matemática do Instituto Superior de Engenharia do Porto \\ ana.meira.castro@eu.ipp.pt \\ António Fiúza \\ Faculdade de Engenharia, Universidade do Porto \\ afiuza@fe.up.pt
}

\begin{abstract}
SUMÁRIO
As práticas agrícolas contemporâneas encontram-se entre as principais fontes antropogénicas de nitrato. Concentrações elevadas de nitratos nos solos e lençóis de água provocam efeitos prejudiciais na saúde humana e criam desequilíbrios substantivos em diversos ecossistemas. De entre diversas abordagens de prevenção e controlo de poluição, as tecnologias de remediação apresentam uma relevância crescente, das quais se destaca as barreiras reativas permeáveis. 0 presente trabalho visa, no contexto das alterações introduzidas pelo crescimento sistemático das concentrações de nitrato em diversos ecossistemas, aquilatar os méritos e limitações relativas das barreiras reativas permeáveis e perspetivar possíveis vias de melhoria do desempenho. Para este efeito, foi conduzida uma pesquisa sistemática em diversas bases-de-dados segundo quatro etapas para identificar literatura relevante. É possível concluir que as barreiras reativas permeáveis constituem-se atualmente como uma tecnologia eficaz de desnitrificação, contudo não foram constatadas evidências da utilização de nanotecnologias para potenciar o desempenho nestes processos de desnitrificação.
\end{abstract}

Palavras-chave: Nitratos, Barreira reativa permeável, saúde ambiental, prevenção e controlo da poluição, nanotecnologia.

\section{Introdução}

O azoto é uma substância essencial para a vida, sendo um elemento constituinte de aminoácidos e outras macromoléculas. Comparativamente com outros elementos essenciais para a vida, como o oxigénio, carbono, fósforo e o enxofre, o azoto é o elemento mais abundante da superfície terrestre, contudo é o menos bio-disponível. A utilização crescente de fertilizantes sintéticos, desde o século XIX, e as práticas intensivas de pecuária têm levado à ocorrência de concentrações excessivas de nitratos, assim como de outras espécies de azoto reativo, em solos e águas subterrâneas em diversas regiões do mundo. Atualmente, o nitrato é considerado o contaminante mais ubíquo em águas subterrâneas à escala planetária (Robertson, 2008), sendo os escoamentos de origem agrícola uma das suas principais causas (Liu, 2013). Os efeitos prejudiciais para o ambiente e os riscos para a saúde humana resultantes de elevadas concentrações de nitratos no solo e águas subterrâneas são atualmente considerados o tema ambiental mais relevante após as emissões de dióxido de carbono. Projeções efetuadas para as próximas décadas deixam antever o acentuar dos atuais efeitos resultantes de elevadas concentrações de nitrato e outras espécies de azoto reativo na biosfera; a utilização de fertilizantes sintéticos e as práticas pecuárias aportam contribuições significativas para essa realidade (Bouwman, 2013). 
O objetivo do presente trabalho é, a partir de uma perspetiva global sobre as implicações para a saúde humana e impacte ambiental decorrentes de elevadas concentrações de nitrato no solo e águas subterrâneas, aquilatar a relevância das tecnologias de remediação num contexto mais amplo da abordagens de prevenção e controlo da poluição causada por nitrato e, para estas tecnologias de remediação, saber quais os respetivos méritos e constrangimentos relativos, muito em particular para as barreiras reativas permeáveis. Para estas últimas pretende-se ainda perspetivar eventuais oportunidades de melhoria de eficácia para processos de remediação de nitrato.

\section{Metodologia}

Para efeito de concretização do objetivo do presente estudo, foram empregues 34 bases-dedados para conduzir uma pesquisa bibliográfica. A pesquisa bibliográfica foi efetuada segundo uma abordagem metodológica estruturada em 4 etapas principais. Na primeira etapa foi utilizado um primeiro conjunto de palavras-chave para a identificação de publicações para análise posterior. Na segunda etapa, e para o mesmo propósito, utilizaram-se sinónimos do primeiro conjunto de palavras-chave. Estas duas etapas iniciais permitiram compilar um conjunto relevante de bibliografia. A Terceira etapa consistiu em pesquisa adicional das basesde-dados, desta vez recorrendo a outras palavras-chave obtidas a partir do primeiro conjunto de elementos bibliográficos selecionados. Na quarta e última etapa, recorreu-se à bibliografia indicada nos artigos selecionados para identificar outras referências bibliográficas relevantes. O conjunto de documentos compilados por este processo foi utilizado para o objetivo deste trabalho.

\section{Resultados}

\section{Efeitos sobre a saúde humana}

No que respeita os efeitos adversos na saúde humana decorrentes da ingestão ou absorção cutânea de nitrato, é consensual a possibilidade do nitrato reagir com aminas e outros compostos contendo azoto, originando nitrosaminas, elementos cancerígenos (Doyle, 1997; Huang, 1998). A ocorrência de nitrato em água para consumo humano constitui uma ameaça para crianças com menos de 6 meses de idade. Uma vez ingerida a água com nitrato, este é reduzido a nitrito $\left(\mathrm{NO}_{2}{ }^{-}\right)$, que por sua vez combinado com a hemoglobina origina metahemoglobina, restringido o transporte de oxigénio no corpo, e causando metahemoglobinemia, condição também designada por “doença do bebé azul” (Kapoor, 1997). O Centro de Clinicas Mayo no Minnesota conduziu pesquisa que demonstrou a correlação entre a ingestão de água doméstica contendo elevadas concentrações de nitrato e uma maior incidência de carcinomas, nomeadamente da tiroide (Ward, 2010), bexiga, estômago e dos ovários (Van Leeuwan, 1999).

\section{Efeitos ambientais}

Os impactes ambientais prejudiciais originados pelo azoto reativo englobam a acidificação de solos e águas superficiais, a eutrofização e a ocorrência de áreas hipóxicas associadas (Hoek, 1997; Horswill, 2008; Long, 2011; Sliggers, 2004), assim como, mudanças na composição e diversidade do biota dos solos (Bradley, 2006; Cairney, 1999), frequentemente conduzindo à perda de biodiversidade e à diminuição da saúde de ecossistemas (Galloway, 2003). Efeitos adversos à escala global incluem o aumento da concentração com gases de efeito de estufa 
(Fowler, 2013; Sliggers, 2004; Zhang, 2011) a depleção da camada de ozono, como resultado das emissões de óxido nitroso (Fowler, 2013; Long, 2011; Zou, 2010) e a inibição da oxidação do metano na atmosfera por nitrato (Mochizuki, 2012).

\section{Abordagens para o controlo da concentração de nitrato no solo e lençóis de água}

Atendendo aos efeitos do nitrato na saúde humana e ambiente, a Comunidade Europeia produziu a Diretiva $n .^{\circ} 91 / 676 / C E E$ que fixou em $50 \mathrm{mg} / \mathrm{l}$ a concentração limite a partir da qual as águas são consideradas poluídas ou em risco de o virem a ser. Também a Organização Mundial de Saúde (OMS) indica 50mg/l como limiar máximo para a concentração de nitrato na água para ingestão. Contudo, para este efeito, a OMS refere também como concentração limite de nitrito na água $3 \mathrm{mg} / \mathrm{l}$, uma outra espécie de azoto reativo. Deste modo, quando ambos nitrato e nitrito ocorrem simultaneamente na água, a soma das proporções da concentração destes compostos face aos respetivos valores limite não deve ultrapassar a unidade.

A Diretiva n. ${ }^{\circ}$ 91/676/CEE estabelece como ações de controlo de concentrações elevadas de nitrato nos lençóis de água a implementação de um código de boas práticas agrícolas, a imposição de regras relativas aos processos de fertilização dos solos e armazenamento de estrume animal. A Declaração de Nanjing sobre a gestão do azoto reativo, para as práticas agrícolas, propõe o incremento da eficácia e eficiência da produção agrícola, enquanto se reduz os efeitos negativos do azoto reativo, e o aumento da disponibilidade do azoto reativo para a produção de alimentos, fibras entre outros em áreas geográficas com insuficiência de azoto, evitando a sua poluição. A este título, Galloway (2008) refere que o aumento da eficiência das plantações na absorção de azoto e a melhoria das estratégias de gestão animal se traduzem, para cada uma, numa redução de $15 \mathrm{Tg} \mathrm{N} \mathrm{a}^{-1}$, tendo por base as $187 \mathrm{Tg} \mathrm{N} \mathrm{a}^{-1}$ produzidas em 2005.

Medidas adicionais de controlo da poluição causada por nitrato envolvem o recurso a remediação do nitrato no solo e lençóis de águas. Tecnologias como a osmose inversa, troca iónica e eletrodiálise têm um historial relevante e bem sucedido, contudo apresentam algumas desvantagem, nomeadamente, não resolvem integralmente o problema decorrente das concentrações elevadas de nitrato, geram efluentes ricos em nitratos - difíceis de descarte adequado, apresentam um nível de complexidade tal que dificulta a sua aplicação in situ, além de que apresentam custos de operação relativamente elevados (Barrabés, 2011, Rocca, 2007). A hidrogenação catalítica de nitratos é também uma tecnologia promissora, no entanto, a formação de amónio é um obstáculo ainda a ultrapassar (Barrabés, 2011).

\section{Barreiras Reativas Permeáveis}

As abordagens mais relevantes para reduzir a concentração de nitrato em água subterrânea são processos de desnitrificação (Bednarek, 2014, Gibert, 2008). Como o nitrato se difunde facilmente para ambientes subsuperficiais, e uma vez aí demonstra ter uma elevada mobilidade nos solos (Moon, 2004), a aplicação de processos de desnitrificação deve ocorrer tão perto da fonte de poluição quanto possível, de modo a limitar os diversos impactes adversos à medida que espécies de azoto reativo se deslocam para jusante nos lençóis de água (Galloway, 2003). A tecnologia das Barreiras Reativas Permeáveis (BRP) é um sistema passivo in situ muito eficaz (Liu, 2013), que possibilita a obtenção do gás azoto a partir de nitrato por intermédio de processos biológicos, (Bednarek, 2010; Schmidt, 2012). A aplicação das BRP é relativamente recente, apresentando aproximadamente 20 anos de existência. As BRP constituídas por fontes 
de carbono orgânico têm demonstrado eficácia na remoção de azoto a custos controlados. Estas estruturas de remediação apresentam ainda a vantagem de serem flexíveis na sua construção, têm um impacto visual mínimo e geralmente longo período de vida útil, ainda que dependente da quantidade de carbono orgânico disponível. Como limitações das BRP refere-se que diferentes fontes de carbono apresentam diferentes eficácias de remediação de nitrato, a eficácia de determinada aplicação de BRP depende das condições hidrológicas locais, níveis freáticos mais baixas favorecem a remediação biótica de nitratos, a redução de nitratos está também dependente de fatores como a temperatura e o oxigénio dissolvido (Bednarek, 2014). A necessidade de um adequado controlo da biomassa pode também constituir-se uma dificuldade (Rocca, 2007). Trabalhos laboratoriais têm empregue processos de desnitrificação associados ao uso de ferro zero valente para o controlo de variáveis biogeoquímicas (Liu, 2013). Falta contudo elucidar qual o impacte na eficácia do desempenho das BRP quando processos de desnitrificação são conjugadas com nanotecnologias.

\section{Conclusões}

As práticas agrícolas contemporâneas, além dos benefícios aportados na provisão de alimentos para uma população mundial em expansão, tem, entre outros efeitos, contribuído para alterações relevantes do ciclo do azoto, em grande medida por via do nitrato. Concentrações elevadas de nitrato nos solos e lençóis de água são reconhecidos como uma ameaça para a saúde humana e o equilíbrio ambiental. De entre as várias medidas de prevenção e controlo da poluição causada por nitrato, atendendo às dinâmicas em curso na utilização de fertilizantes e práticas pecuárias, haverá nas próximas décadas um acentuar da dependência da humanidade de tecnologias de remediação de nitrato. A tecnologia das BRP tem demonstrado eficácia no controlo de risco para a saúde ambiental resultante de concentrações elevadas de nitrato no solo e águas subterrâneas. No contexto atual e para o futuro que se perspetiva, o aumento da eficácia da tecnologia das BRP parece ser uma necessidade. A conjugação de nanotechnologias com processos de desnitrificação poderá eventualmente aportar uma eficácia acrescida às BRP.

\section{Bibliografia:}

Barrabés, N., J. Sá, Catalytic nitrate removal from water, past, present and future perspectives. Applied Catalysis B: Environmental, 2011, (104), 1- 5.

Bednarek, A., S. Szklarek, M., Zalewski, M., Nitrogen pollution removal from areas of intensive farmingcomparison of various denitrification biotechnologies. Ecohydrology \& Hydrobiology, 2014, (14), 132 141.

Bednarek, A., Stolarska, M., Ubraniak, M., Zalewski, M. Application of permeable reactive barrier for reduction of nitrogen load in the agricultural areas - preliminary results. Ecohydrology \& Hydrobiology, 2010, (2-4), 355-362.

Bradley, K., Rhae A. Drijber, J. Knops, Increased $\mathrm{N}$ availability in grassland soils modifies their microbial communities and decreases the abundance of arbuscular mycorrhizal fungi. Soil Biology \& Biochemistry, 2006, (38), 1583-1595.

Bouwman, L., K.K. Goldewijk, K.W. Van Der Hoek, A.H. W. Beusen, D.P. Van Vuuren, J. Willems, M.C. Rufino, E. Stehfest, Exploring global changes in nitrogen and phosphorus cycles in agriculture induced by livestock production over the 1900-2050 period. PNAS, 2013, 110(52), 20882-20887.

Cairney, J. W. G., A.A. Meharg, Influences of anthropogenic pollution on mycorrhizal fungal communities. Environmental Pollution, 1999, (106), 169-182. 
Doyle, M. P., Beuchat, L.R., Montville, T.J., Food Microbiology Fundamentals and Frontiers. Edtion ed. Washington, DC: ASM Press, 1997.

Fowler, D., M. Coyle, U. Skiba, M.A. Sutton, J. N. Cape, S. Reis, L.J. Sheppard, A. Jenkins, B. Grizzetti, J.N. Galloway, P. Vitousek, A. Leach, A.F. Bouwman, K. Butterbach-Bahl, F. Dentener, D. Stevenson, M. Amann, M. Voss, The global nitrogen cycle in the twentyfirst century. Philosophical Transactions of the Royal Society, 2013, (29), 1-13.

Gibert, O., S. Pomierny, I. Rowe, R.M. Kalin, Selection of organic substrates as potential reactive materials for use in a denitrification permeable reactive barrier (PRB). Bioresource Technology, 2008, (99), 7587-7596.

Hoek, A., Scenarios of Animal Waste Production and Fertilizer Use and Associated Ammonia Emission for the Developing Countries. Atmospheric Environment, 1997, 24(31), 4095-4102.

Horswill, P., O. O'Sullivan, G.K. Phoenix, et al., Base cation depletion, eutrophication and acidification of species-rich grasslands in response to long-term simulated nitrogen deposition. Environmental Pollution, 2008, (155), 336-349.

Huang, C.-P., H.-W. Wang, P.-C. Chiu, Nitrate Reduction by Metallic Iron. Water Research, 1998, 32(8), $2257-$ 2264.

Kapoor, A., Viraraghavan, T., Nitrate removal from drinking water-review. Journal of Environmental Engineering, 1997, (123), 371-380.

Liu, S.-J., Z.-Y. Zhao, J. Li, J. Wang, Y. Qi, An anaerobic two-layer permeable reactive biobarrier for the remediation of nitrate-contaminated groundwater. Water Research, 2013, (47), 5977-5985.

Long, L.M., L.A. Schipper, D.A. Bruesewitz, Long-term nitrate removal in a denitrification wall. Agriculture, Ecosystems and Environment, 2011, (140), 2011.

Mochizuki, Y., K. Koba, M. Yoh, Strong inhibitory effect of nitrate on atmospheric methane oxidation in forest soils. Soil Biology \& Biochemistry, 2012, 50(164-166).

Moon, H.S., K.-H. Ahn, S. Leeb, K. Nama, J.Y. Kim, Use of autotrophic sulfur-oxidizers to remove nitrate from bank filtrate in a permeable reactive barrier system. Environmental Pollution, 2004, (129), 499-507.

Robertson, W. D., J.L. Vogan, P.S. Lombardo, Nitrate Removal Rates in a 15-Year-Old Permeable Reactive Barrier Treating Septic System Nitrate. Groundwater monitoring and remediation, 2008, 28(3), 6572.

Rocca, C. D., Vincenzo Belgiorno, Sureyya Meriç, Overview of in-situ applicable nitrate removal processes. Desalination, 2007, (204), 46- 62.

Schmidt, C.A., M.W. Clark, Efficacy of a denitrification wall to treat continuously high nitrate loads. Ecological Engineering, 2012, (42), 203- 211.

Sliggers, J. The need for more integrated policy for air quality, acidification and climate change: reactive nitrogen links them all. Environmental Science \& Policy, 2004, (7), 47-58.

Van Leeuwan, J. A., Walter-Toews, D., Abernathy, T., Smit, B., Shoukri, M., Associations between stomach cancer incidence and drinking water contamination with atrazine and nitrate in Ontario (Canada) agroecosystems, 1987-1991. International Journal of Epidemiology, 1999, (28), 836-840.

Ward, M.H., Kilfoy, Briseis A., Weyer, P.J., Anderson, Kristin E., Folsom, Aaron R., Cerhan, James R., Nitrate Intake and the Risk of Thyroid Cancer and Thyroid Disease. Epidemiology, 2010, 21(3), 389-395.

Zhang, X. L. A. F., Nitrogen fertilizer induced greenhouse gas emissions in China. Current Opinion in Environmental Sustainability, 2011, (3), 407-413.

Zou, J., Yanyu Lu, Yao Huang, Estimates of synthetic fertilizer $\mathrm{N}$-induced direct nitrous oxide emission from Chinese croplands during 1980-2000. Environmental Pollution, 2010, (158), 631-635. 